\title{
CONTINUIDADE ESPACIAL DE ATRIBUTOS FÍSICO-HÍDRICOS DO SOLO EM SUB-BACIA HIDROGRÁFICA DE CABECEIRA ${ }^{1}$
}

\author{
Spatial Continuity of Soil Physical-Hydric Attributes at Headwater Watershed
}

\author{
José Alves Junqueira Júnior², Antonio Marciano da Silva³, Carlos Rogério de Mello ${ }^{4}$, Daniel Brasil Ferreira Pinto
}

\begin{abstract}
RESUMO
O conhecimento da estrutura de continuidade espacial dos atributos fisico-hídricos de solos é uma importante ferramenta para o manejo solo em sub-bacias hidrográficas. No entanto, o mapeamento produzido não pode apresentar-se enviesado, com tendências, como acontece com aqueles puramente geométricos. Desta forma, neste trabalho, objetivou-se avaliar modelos de semivariogramas, bem como, métodos de ajuste, para volume total de poros (VTP), condutividade hidráulica do solo saturado $\left(\mathrm{k}_{\mathrm{o}}\right)$, porosidade drenável $(\mu)$, umidade volumétrica na capacidade de campo $\left(\theta_{c c}\right)$, umidade volumétrica no ponto de murcha permanente $\left(\theta_{\text {pmp }}\right)$ e capacidade total de armazenamento de água (CTA), em uma sub-bacia hidrográfica na cabeceira do Rio Grande, na Serra da Mantiqueira. Para isto, foram feitas amostragens em 198 pontos na camada de $0-0,15 \mathrm{~m}$ em grids de $300 \mathrm{~m}$ x 300m, 60m x 60m e 20m x 20m. Foram ajustados os modelos de semivariogramas esférico, exponencial e gaussiano ao semivariograma experimental, pelos métodos dos mínimos quadrados ponderados (MQP) e máxima verossimilhança (MV). A análise variográfica mostrou que todos os atributos estudados apresentaram-se estruturados espacialmente. A maioria dos atributos apresentou melhor ajuste pela metodologia dos MQP, exceção feita aos atributos porosidade drenável e $\theta_{\mathrm{pmp}}$. Não houve predominância de nenhum modelo específico de semivariogramas para os atributos avaliados.
\end{abstract}

Termos para indexação: validação cruzada, mínimos quadrados ponderados, máxima verossimilhança.

\section{ABSTRACT}

The knowledge of spatial continuity of soil physical-hydric attributes is an important tool for soil management on watersheds. However, the mapping cannot present bias as produced by essential geometric interpolator. This work aims to evaluate semivariogram models and respective methods of adjustment for total porosity (VTP), hydraulic conductivity saturated ( $\mathrm{k}_{\mathrm{o}}$ ), drainable porosity $(\mu)$, soil moisture in field capacity $\left(\theta_{\mathrm{cc}}\right)$, soil moisture in permanent wilt point $\left(\theta_{\mathrm{pmp}}\right)$ and soil water storage capacity (CTA) on headwater watershed, in Mantiqueira Mountain region. For that, samples were collected at layer of 0-0.15 m, in 198 points, following the grids of $300 \mathrm{~m} \times 300 \mathrm{~m}, 60 \mathrm{~m} \times 60 \mathrm{~m}$ and $20 \mathrm{~m}$ x $20 \mathrm{~m}$. Weighted minimum squared (MQP) and Maximum Likelihood (MV) methods were used to adjust spherical, exponential and gaussian semi-variogram models. Cross validation showed that all attributes studied presented spatial continuity. Most of soil attributes presented better adjustments through MQP methodology, except drainable porosity and $\theta_{\mathrm{pmp}}$. It was not possible to determine only one semi-variogram model to all soil attributes evaluated.

Index terms: Cross validation, weighted minimum squared, maximum likelihood.

\section{(Recebido em 21 de setembro de 2006 e aprovado em 13 de setembro de 2007)}

\section{INTRODUÇÃO}

Compreender a distribuição espacial de fenômenos naturais constitui-se em um desafio para a elucidação de questões importantes em diversas áreas do conhecimento. $\mathrm{O}$ avanço do conhecimento científico tem evidenciado as limitações dos métodos tradicionais da estatística no tratamento da variabilidade espacial de variáveis físicohídricas do solo. As técnicas da estatística clássica partem do pressuposto de que todas as amostras são aleatórias e sua aplicação não envolve qualquer conhecimento da posição atual das mesmas ou do relacionamento entre essas posições. Já a geoestatística tem como pressuposto que as propriedades naturais da superfície terrestre são espacialmente contínuas, necessitando-se da aplicação de preceitos importantes associados à dependência espacial das variáveis continuamente distribuídas no espaço (CRESSIE, 1993).

O semivariograma é a ferramenta que possibilita o estudo da dependência espacial entre amostras num campo experimental e define os parâmetros necessários para realização da krigagem (BRUNDSDON et al., 1996). Segundo

\footnotetext{
${ }^{1}$ Com apoio da CEMIG/ANEEL, CNPq e FAPEMIG

${ }^{2}$ Engenheiro Agrícola, Mestre em Engenharia Agrícola - Centro Federal de Educação Tecnológica de Cuiabá/CEFET - Br 364, Km 329 - São Vicente da Serra - 78106-000 - Santo Antônio do Leverger, MT - junqueira@yahoo.com.br

${ }^{3}$ Engenheiro Agrônomo, Doutor em Hidráulica e Saneamento, Professor Titular - Departamento de Engenharia/DEG - Universidade Federal de Lavras/ UFLA - Cx. P. 3037 - 37200-000, Lavras, MG - marciano@ufla.br - Bolsista CNPq

${ }^{4}$ Engenheiro Agrícola, Doutor em Ciência do Solo, Professor Adjunto - Departamento de Engenharia/DEG - Universidade Federal de Lavras/UFLA - Cx. P. 3037 - 37200-000, Lavras, MG - crmello@ufla.br - Bolsista CNPq

${ }^{5}$ Engenheiro Agrícola, Doutorando - Departamento de Engenharia/DEG - Universidade Federal de Lavras/UFLA - Cx. P. 3037 - 37200-000, Lavras, MG brasil_ufla@yahoo.com.br - Bolsista CNPq
}

Ciênc. agrotec., Lavras, v. 32, n. 3, p. 914-922, maio/jun., 2008 
Lamparelli et al. (2001), o semivariograma é uma ferramenta básica que permite descrever quantitativamente a variação no espaço de um fenômeno regionalizado. Existem vários métodos de ajuste de semivariogramas, destacando-se os Métodos dos Mínimos Quadrados Ordinários (MQO), Métodos dos Mínimos Quadrados Ponderados (MQP) e Máxima Verossimilhança (MELLO et al., 2005).

Gomes (2005), trabalhando com atributos físicohídricos do solo numa sub-bacia hidrográfica representativa dos Latossolos, relata que os melhores resultados de ajuste de semivariogramas foram obtidos pelo método da máxima verossimilhança (MV), especialmente capacidade de retenção de água e condutividade hidráulica. Já Vieira (2005) obteve melhor modelagem do semivariograma pelo método MQP para uma série de atributos físico-hídricos do solo numa microbacia hidrográfica da região de Lavras, constituída basicamente por Cambissolos.

Neste contexto, este trabalho objetiva estudar a estrutura de continuidade espacial de alguns atributos físicohídricos do solo, analisando o grau de dependência espacial e procedimentos para modelagem do semivariograma experimental, notadamente os modelos esférico, exponencial e gaussiano e as metodologias da Máxima Verossimilhança (MV) e Mínimos Quadrados Ponderados (MQP).

\section{MATERIAL E MÉTODOS}

O Cambissolo é a unidade pedológica predominante na sub-bacia hidrográfica, havendo, contudo, considerável presença de Neossolos Flúvicos. Na Tabela 1 apresentase a distribuição dos solos da Sub-bacia Hidrográfica do Ribeirão Lavrinhas, na região da Serra da Mantiqueira.

Os atributos físico-hídricos do solo estudados são: o volume total de poros (VTP), umidade volumétrica do solo à capacidade de campo $\left(\theta_{c c}\right)$, porosidade drenável $(\mu)$, umidade volumétrica do solo correspondente ao ponto de murcha permanente $\left(\theta_{\text {PMP }}\right)$ e capacidade total de retenção de água (CTA). O VTP foi calculado com base nas densidades do solo e de partícula, ou seja:

$$
\mathrm{VTP}=\left(1-\frac{\mathrm{D}_{\mathrm{s}}}{\mathrm{D}_{\mathrm{p}}}\right) \cdot 100
$$

Em que VTP é o Volume Total de Poros (\%), $\mathrm{D}_{\mathrm{s}}$ é a densidade do solo $\left(\mathrm{kg} \mathrm{dm}^{-3}\right)$ e $\mathrm{D}_{\mathrm{p}}$ é a densidade de partículas $\left(\mathrm{kg} \mathrm{dm}^{-3}\right)$.

As umidades à capacidade de campo e ponto de murcha permanente foram consideradas como sendo correspondentes às tensões matriciais de $10 \mathrm{kPa}$ e 1500 $\mathrm{kPa}$, respectivamente, segundo Gomes (2005) e Medina \& Oliveira Júnior (1987). A porosidade drenável foi obtida segundo Mello et al. (2002), como sendo a diferença entre as umidades de saturação e capacidade de campo. A CTA foi obtida segundo Bernardo (2005), considerando a diferença entre as umidades à capacidade de campo e ponto de murcha permanente e a profundidade trabalhada (150 mm). A condutividade hidráulica saturada foi determinada utilizando um permeâmetro de fluxo constante, especificamente o permeâmetro de Guelph, modelo 2800K1, com cargas hidráulicas constantes de 0,05 e 0,10 m, seguindo instruções de Gomes (2005).

A amostragem de solo e os testes de condutividade hidráulica foram realizados na camada de 0 a $0,15 \mathrm{~m}$ de profundidade, seguindo um grid regular de $300 \times 300 \mathrm{~m}$ com refinamento da escala em grid's de $60 \times 60$ e $20 \times 20$, perfazendo um total de 198 pontos.

A análise exploratória dos dados incluiu a estatística clássica com a determinação do coeficiente de variação (C.V.), coeficiente de assimetria (C.A.), média, mediana, desvio padrão e teste de normalidade de Shapiro-Wilk a 5

Tabela 1 - Ocorrência das unidades pedológica na sub-bacia hidrográfica Ribeirão Lavrinha.

\begin{tabular}{lrr}
\multicolumn{1}{c}{ Unidades pedológicas } & Área (ha) & Área (\%) \\
\hline CH média pouco profundo & 49,2 & 7,2 \\
CX média A moderado muito profundo & 53,7 & 7,8 \\
CX argilosa A proeminente endopedregoso pouco profundo & 5,5 & 0,8 \\
CX média A moderado endopedregoso pouco profundo & 81,4 & 11,8 \\
CX média A proeminente endopedregoso pouco profundo & 410,8 & 59,8 \\
CX média A proeminente muito profundo & 43,7 & 6,4 \\
NF média A moderado com mosqueados & 22,7 & 3,3 \\
NF média A proeminente com mosqueados & 20,1 & 2,9 \\
\hline Total & 687,1 & 100,0 \\
\hline
\end{tabular}

$\mathrm{CH}$ = Cambissolo Húmico, $\mathrm{CX}=$ Cambissolo Háplico, NF = Neossolo Flúvico. 
\% de significância. A análise exploratória contou ainda com a visualização dos dados em mapas para verificação de tendências e histogramas de freqüência da diferença entre pares de pontos, além de "boxplot" para a identificação de observações atípicas (outliers).

A avaliação do desempenho de cada modelo deuse mediante dois critérios. Avaliou-se a razão entre a variância estrutural $(\mathrm{C})$ e o patamar $\left(\mathrm{C}_{0}+\mathrm{C}\right)$, expressa em porcentagem, pelo grau de dependência espacial (GD). Segundo Zimback (2001), o GD é dado por:

$$
\mathrm{GD}=\left(\frac{\mathrm{C}}{\mathrm{C}+\mathrm{C}_{0}}\right) \cdot 100
$$

Sendo que o GD é considerado fraco se essa razão for $\leq 25 \%$, moderado quando a razão estiver entre $25 \%$ e $75 \%$, e forte se a razão for $>75 \%$.

O outro critério de comparação do desempenho foi o procedimento de validação cruzada, seguindo as recomendações de Cressie (1993) e Mello et al. (2005).

A comparação entre os modelos, em cada método de ajuste, foi efetuada pelo erro médio reduzido ( $\overline{\mathrm{ER}})$, desvio padrão dos erros reduzidos $\left(\mathrm{S}_{\mathrm{ER}}\right)$ e do somatório do quadrado médio do erro ( $\Sigma$ QME ). Segundo Cressie (1993) e McBratney \& Webster (1986), o erro médio reduzido é definido como:

$$
\overline{\mathrm{ER}}=\frac{1}{\mathrm{n}} \sum_{\mathrm{i}=1}^{\mathrm{n}} \frac{\mathrm{z}\left(\mathrm{x}_{\mathrm{io}}\right)-\hat{\mathrm{z}}\left(\mathrm{x}_{\mathrm{io}}\right)}{\sigma\left(\mathrm{x}_{\mathrm{io}}\right)}
$$

Em que $z\left(\mathrm{x}_{\mathrm{io}}\right)$ é o valor observado no ponto i0; $\hat{z}\left(\mathrm{x}_{\mathrm{io}}\right)$ é o valor estimado para o ponto i0 e $\sigma\left(\mathrm{x}_{\mathrm{io}}\right)$ é o desvio padrão da krigagem no ponto i0. O desvio padrão dos erros reduzidos foi obtido a partir da seguinte equação:

$$
S_{E R}=\sqrt{\frac{1}{n} \sum_{i=1}^{n}\left(\left\{\frac{z\left(x_{i o}\right)-\hat{z}\left(x_{i o}\right)}{\sigma\left(x_{i o}\right)}\right\}\right)^{2}}
$$

O erro reduzido médio mais próximo de zero e o desvio padrão próximo da unidade são os critérios para escolha do melhor modelo, além do menor somatório do quadrado médio do erro, representado pela equação 5 .

$$
\sum Q M E=\left(\frac{\sum_{i=1}^{n}\left[z\left(x_{i o}\right)-\hat{z}\left(x_{i o}\right)\right]^{2}}{n}\right)
$$

Todas as análises efetuadas no presente trabalho foram realizadas com o programa $\mathrm{R}$, utilizando-se pacote GeoR (RIBEIRO JÚNIOR \& DIGGLE, 2001).

\section{RESULTADOS E DISCUSSÃO}

Detecta-se pequena variabilidade dos dados, representada pelos relativamente baixos coeficientes de variação, exceto para o atributo $\mathrm{k}_{\mathrm{o}}$. Esta variabilidade pode ser classificada de acordo com os critérios propostos por Warrick \& Nielsen (1980), que consideram os valores do coeficiente de variação menores que $12 \%$ como baixa variabilidade, de $12 \%$ a $60 \%$ média variabilidade e os valores acima de $60 \%$ alta variabilidade. Assim, os atributos VTP, $\theta_{c c}, \mu, \theta_{\text {pmp }}$ e CTA apresentaram média variabilidade e $\mathrm{k}_{\mathrm{o}}$, alta variabilidade. A alta variabilidade encontrada para $\mathrm{k}_{0}$ está em consonância com o comportamento do histograma de frequiência da diferença entre pares de pontos para este atributo (Figura 1b) e com o alto coeficiente de assimetria comparando-se com os demais atributos. A semelhança entre as medidas de posição (média e mediana) dos atributos, também expressa simetria na distribuição dos dados.

Verifica-se pelo teste de normalidade de ShapiroWilk, que os atributos $\mathrm{k}_{\mathrm{o}}, \theta_{\text {pmp }}$ e $\mu$ não apresentaram distribuição normal, uma vez que os valores da estatística p apresentaram-se altamente significativos, aceitando-se a hipótese $\mathrm{H}_{\mathrm{o}}$ de não normalidade. Este comportamento pode ser mais bem interpretado observando o comportamento dos histogramas das diferenças entre pares de pontos apresentados pela Figura 1, principalmente para $\mathrm{o}$ atributo $\mathrm{k}_{\mathrm{o}}$. Assim, é possível que haja dificuldades no ajuste dos semivariogramas pela metodologia da máxima verossimilhança, devido ao fato de o ajuste do modelo de semivariograma por esta metodologia não se basear nos pontos do semivariograma e, sim, nas diferenças dos dados originais (DIGGLE \& RIBEIRO JUNIOR, 2007).

Os demais atributos apresentaram valores de p não significativos a $1 \%$, ou seja, apresentaram distribuição simétrica com tendência à normalidade, rejeitando-se, portanto, a hipótese $\mathrm{H}_{\mathrm{o}}$. Este fato também pode ser constatado pela semelhança entre as medidas de posição (média e Mediana) dos atributos.

$\mathrm{Na}$ Tabela 2 estão apresentados os resultados referentes à estatística clássica para cada atributo físicohídrico avaliado.

$\mathrm{O}$ atributo $\mathrm{k}_{\mathrm{o}}$ (Figura $1 \mathrm{~b}$ ) apresentou o maior número de "outliers", sete no total. Este fato também foi observado por Gomes (2005), o qual relata que tal atributo apresenta grande variabilidade espacial e estrutura de dependência espacial indefinida. É possível observar que, mesmo após a remoção dos "outliers", este atributo apresentou grande distorção em seu gráfico "boxplot” e distribuição 
assimétrica, com comportamento do histograma da diferença entre pares de pontos, indefinido, o que pode ser um indício de maior dificuldade no ajuste de semivariogramas, principalmente pelo método da máxima verossimilhança.

A análise exploratória dos atributos físico-hídricos está representada nas Figuras 1, 2 e 3. Inicialmente observase uma boa distribuição espacial dos dados em todas as direções da sub-bacia hidrográfica, ou seja, não existe nenhum tipo de tendenciosidade direcionada. A existência de tendências pode inviabilizar a aplicação da geoestatística, uma vez que a condição de não tendenciosidade é de fundamental importância, sendo exigida para aplicação da geoestatística.

A análise exploratória permitiu detectar que houve valores candidatos a "outliers" para dois atributos analisados $\left(\mathrm{k}_{\mathrm{o}} \mathrm{e} \mu\right)$. Estes pontos produzem maior variabilidade dos dados e maior dispersão dos mesmos em torno da média, prejudicando a normalidade. Quando detectados pelos gráficos "boxplot", estes valores foram comparados com seus vizinhos mais próximos, seguindo as recomendações de Libardi \& Melo Filho (2006), e foram retirados quando apresentaram diferenças acentuadas. Desta forma, os novos gráficos são apresentados sem a presença destes, exceção feita ao atributo $\mu$ que não teve seu "outlier" retirado porque este ponto não apresentou diferenças acentuadas com seus vizinhos.

Os demais atributos avaliados $\left(\theta_{c c}\right.$, CTA, $\theta_{\text {pmp }}$ e VTP), não apresentaram valores discrepantes, notando-se boa distribuição de frequiências em termos de normalidade e do gráfico "boxplot" para os atributos $\theta_{\text {cc }}$, CTA, e VTP e uma ligeira distorção para $\theta_{\mathrm{pmp}}$.

Percebe-se que alguns atributos não se ajustaram por MV, caso específico de CTA, VTP e $\theta_{\text {cc }}$. Os demais atributos $\left(\theta_{\text {pmp }}, \mathrm{k}_{\mathrm{o}}, \mu\right)$ se ajustaram por ambas as metodologias.

Analisando os semivariogramas visualmente, notase grande semelhança em seus comportamentos, principalmente aqueles ajustados por MQP, sendo muito difícil se determinar qual o melhor modelo para cada atributo avaliado.
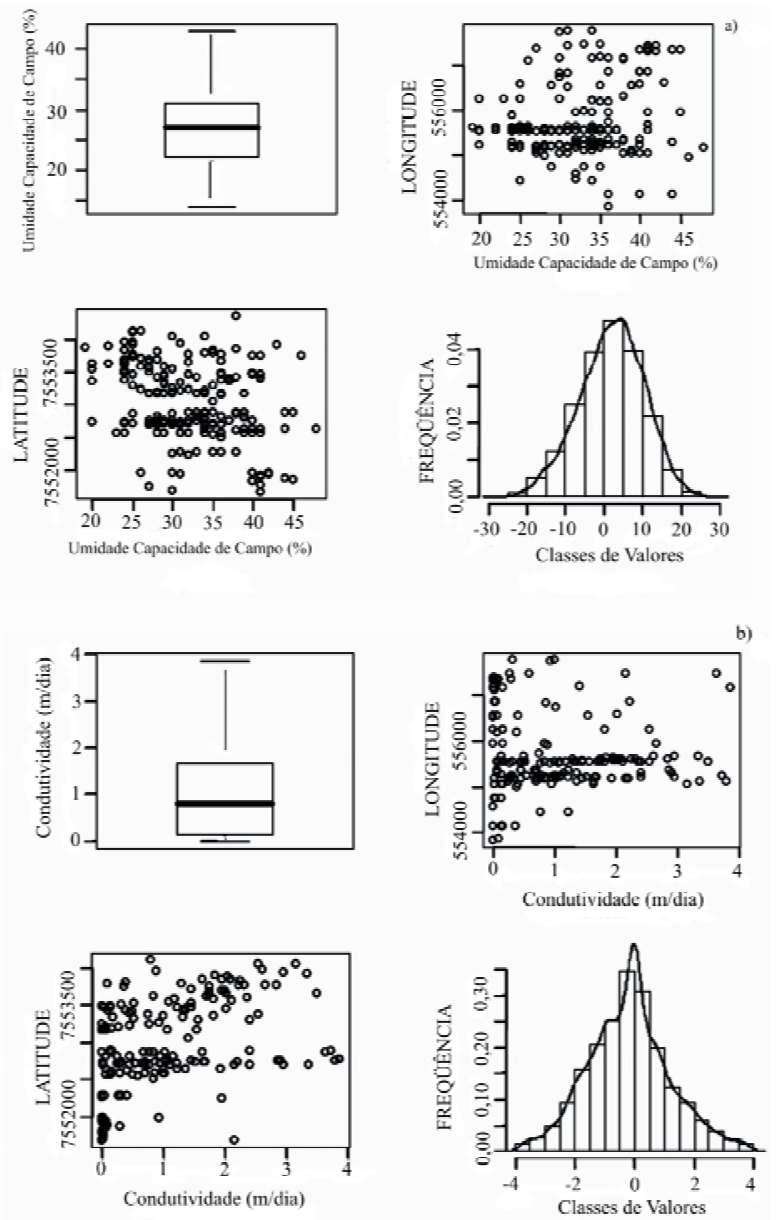

Figura 1 - Gráficos do tipo boxplot, mapas de tendência e histograma das diferenças entre pares de pontos para os atributos umidade na capacidade de campo (a) e condutividade hidráulica (b).

Tabela 2 - Parâmetros estatísticos dos atributos físico-hídricos estudados.

\begin{tabular}{cccccc}
\hline Variável & $\bar{x}$ & Mediana & C.V.(\%) & C.A. & $\mathrm{p}<0,05$ \\
\hline$\theta_{\mathrm{cc}}\left(\mathrm{cm}^{3} \mathrm{~cm}^{-3}\right)$ & 0,325 & 0,319 & 20,0 & 0,409 & $0,0352^{\text {ns }}$ \\
$\mathrm{k}_{\mathrm{o}}\left(\mathrm{m} \mathrm{dia}^{-1}\right)$ & 1,69 & 1,01 & 180,7 & 6,692 & $2,436 \mathrm{E}-10^{*}$ \\
CTA $(\mathrm{mm})$ & 23,56 & 23,97 & 41,5 & $-0,039$ & $0,0377^{\text {ns }}$ \\
$\theta_{\text {pmp }}\left(\mathrm{cm}^{3} \mathrm{~cm}^{-3}\right)$ & 0,2000 & 0,167 & 35,4 & 0,548 & $0,002325^{*}$ \\
$\mu(\%)$ & 26,96 & 27,14 & 42,5 & 0,329 & $0,0267^{\text {ns }}$ \\
VTP $(\%)$ & 59,44 & 58,93 & 13,2 & 0,100 & $0,0006297^{*}$ \\
\hline
\end{tabular}

${ }^{*}=$ significativo a $1 \%,{ }^{\mathrm{ns}}=$ não significativo a $1 \%$. 

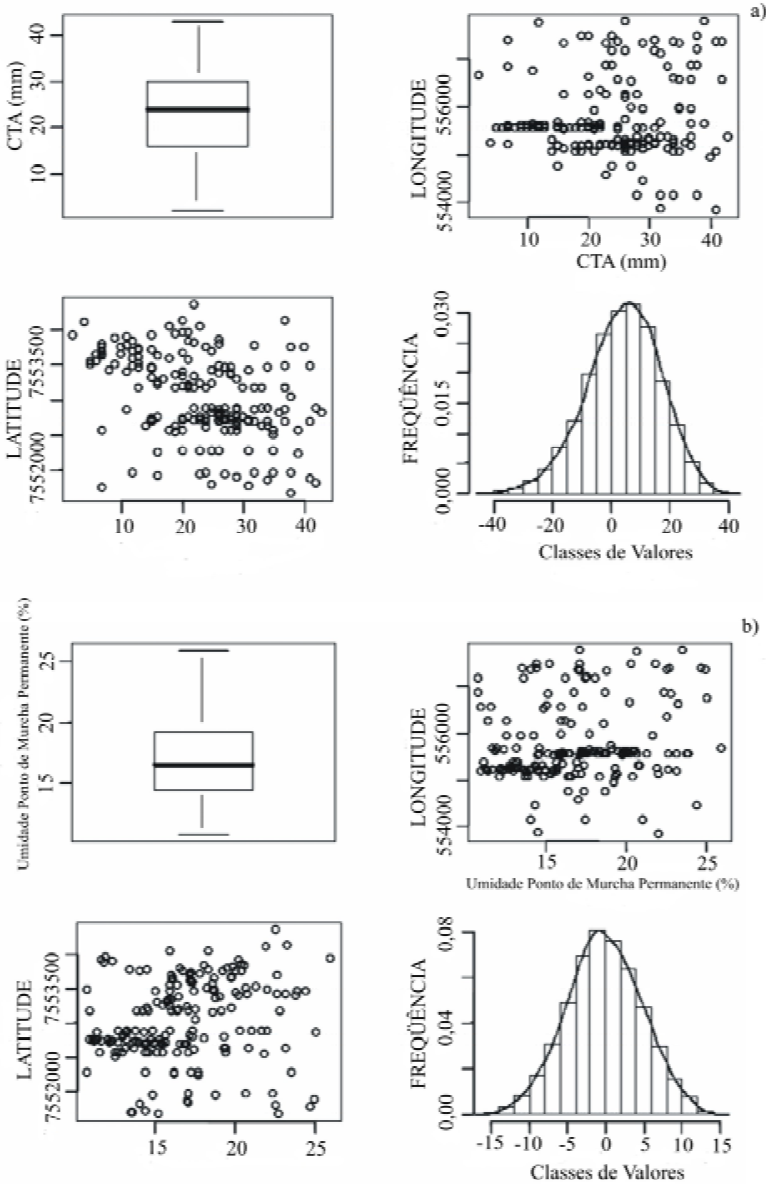

Figura 2 - Gráficos do tipo boxplot, mapas de tendência e histograma das diferenças entre pares de pontos para os atributos CTA (a) e umidade no ponto de murcha permanente (b).

Percebe-se que em toda a extensão do semivariograma as curvas mantêm-se próximas, chegando a se tangenciarem em alguns casos, apresentando comportamento semelhante. É interessante destacar que o atributo $\mu$ apresenta comportamento diferenciado em relação aos demais, pois é o único atributo que apresenta similaridade no comportamento dos semivariogramas ajustados por ambas as metodologias. Esta mesma análise para os modelos ajustados por MV revela também que há semelhança entre os modelos, mais não tão pronunciada como o ajuste por MQP. Para o atributo $\theta_{\text {pmp }}$, a partir da distância de $750 \mathrm{~m}$ os modelos exponencial e gaussiano ajustados por MV apresentam comportamento semelhante, com as curvas mantendo-se próximas, mas, não se tangenciando. Já para o atributo $\mathrm{k}_{\mathrm{o}}$, os modelos esférico e gaussiano ajustados por MV são os que mais se assemelham, sugerindo que para estes atributos deve haver uma melhor metodologia, a partir da qual se produzirá um mapa de krigagem mais consistente, representativo da realidade físico-hídrica da sub-bacia hidrográfica.

$\mathrm{Na}$ Figura 4 apresentam-se os modelos de semivariogramas ajustados pelas metodologias empregadas, para os atributos físico-hídricos estudados na sub-bacia hidrográfica.
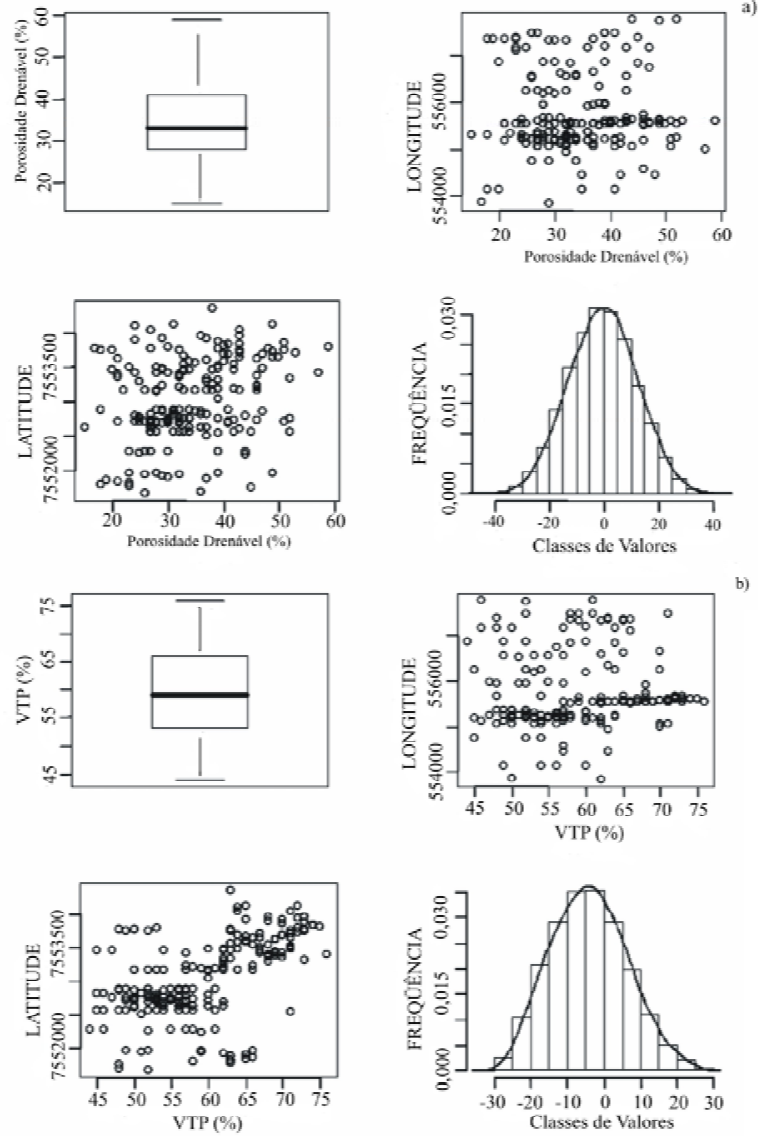

Figura 3 - Gráficos do tipo boxplot, mapas de tendência e histograma das diferenças entre pares de pontos para os atributos porosidade drenável (a) e VTP (b).

Encontram-se na Tabela 3 os parâmetros dos semivariogramas ajustados com base em cada uma das metodologias empregadas. Desta forma, pôde-se observar que para os atributos $\left(\mathrm{k}_{\mathrm{o}}, \theta_{\mathrm{pmp}}\right.$ e $\left.\mu\right)$, cujo ajuste se deu por ambas as metodologias, não houve grandes discrepâncias em relação ao GD, principalmente para $\mathrm{k}_{\mathrm{o}}$ que apresentou, segundo Zimback (2001), moderada dependência espacial, ficando a escolha do método de ajuste e modelo de semivariograma dependente da validação cruzada. 

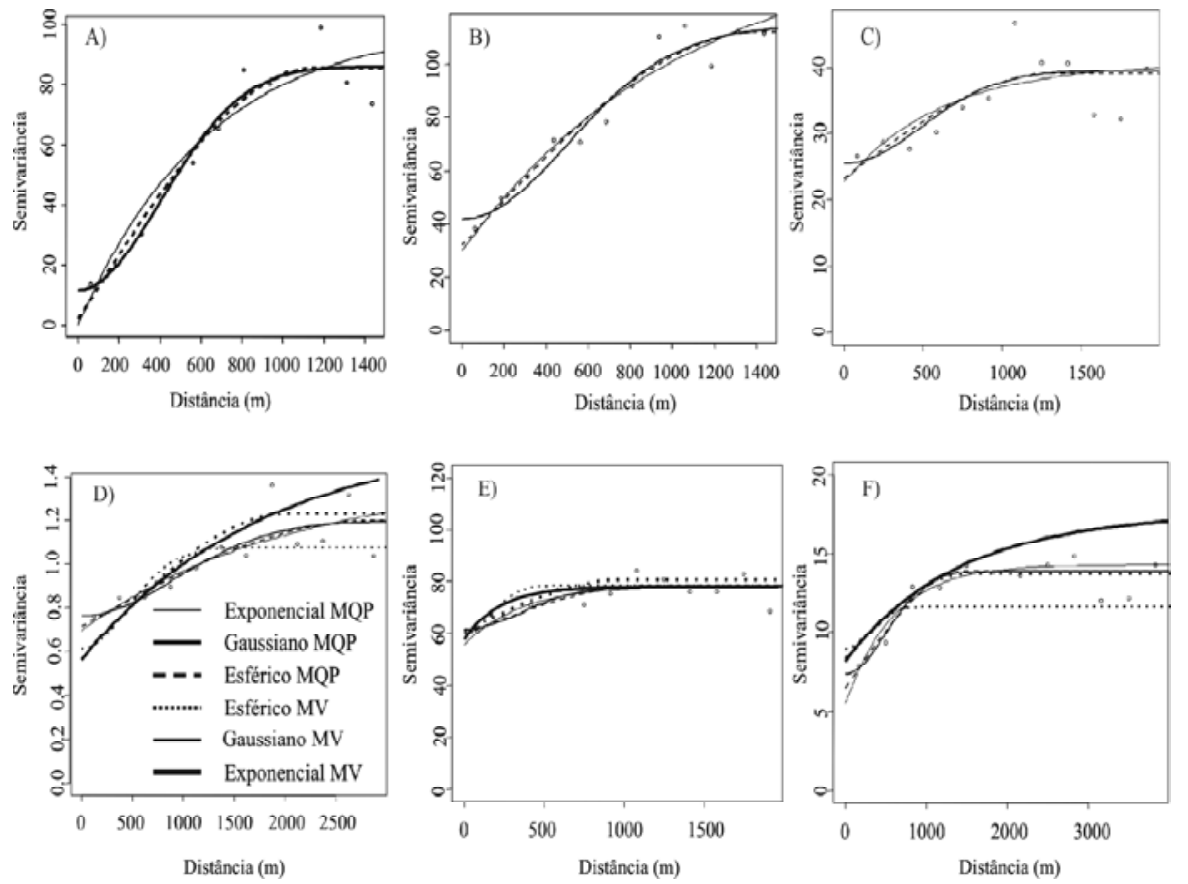

Figura 4 - Semivariogramas ajustados para os atributos físico-hídricos na sub-bacia hidrográfica do Ribeirão Lavrinhas $\left(\mathrm{a}-\mathrm{VTP} ; \mathrm{b}-\mathrm{CTA} ; \mathrm{c}-\theta_{\mathrm{CC}} ; \mathrm{d}-\mathrm{ko} ; \mathrm{e}-\mu ; \mathrm{f}-\theta_{\mathrm{PMP}}\right)$.

Para o atributo $\theta_{\text {pmp }}$, o modelo exponencial por MV apresentou alto grau de dependência espacial e menor efeito pepita, sendo possivelmente o melhor modelo. Para o atributo $\mu$, sobressaiu-se a metodologia dos MQP apresentando moderado grau de dependência espacial para os modelos esférico e exponencial, este apresentando ligeira superioridade sobre o primeiro por causa do menor efeito pepita. Os demais atributos $\left(\theta_{c c}\right.$, CTA e VTP) apresentaram ajuste apenas por MQP. Este melhor desempenho da metodologia MQP pode ser atribuído à falta de normalidade bivariada, como é o caso do atributo VTP, que só apresentou ajuste por esta metodologia. É importante destacar que os atributos $\mathrm{k}_{\mathrm{o}}$ e $\theta_{\text {pmp }}$, apesar de não terem apresentado normalidade dos dados, apresentaram ajuste por MV.

A superioridade da metodologia MQP ajustada a atributos do solo em sub-bacia hidrográfica também foi constatada por Gomes (2005) e Vieira (2005). Tomando-se como referência os valores do GD sugeridos por Zimback (2001), conclui-se que o atributo CTA ajustado por MQP apresentou um forte grau de dependência para o modelo exponencial e moderado para os demais modelos. $\mathrm{O}$ atributo $\theta_{\text {cc }}$ apresentou moderado grau de dependência espacial para todos os modelos ajustados, com pequena magnitude de variação no efeito pepita. Já para o atributo VTP, o GD foi considerado forte para os modelos esférico e exponencial e moderado para o modelo gaussiano. Notase que o alcance dos semivariogramas apresentou valores variando de 53 a 2798 m, compatíveis com a dimensão da sub-bacia e com a maior distância de separação entre os pontos amostrados que foi de $4200 \mathrm{~m}$.

Segundo McBratney \& Webster (1986) e Mello et al. (2005), a comparação entre modelos de semivariogramas ajustados por diferentes metodologias pode ser efetuada através do erro médio reduzido ( $\overline{\mathrm{ER}}$ ), desvio padrão dos erros reduzidos $\left(S_{E R}\right)$ e do somatório do quadrado médio do erro ( $\Sigma \mathrm{QME}$ ).

Visualiza-se na Tabela 4, os valores referentes a estes parâmetros gerados pela validação cruzada. Inicialmente, o que chama a atenção é a grandeza do desvio padrão dos erros padronizados dos atributos VTP e CTA quando comparada à dos demais atributos, refletindo a alta variabilidade destes atributos. Comportamento semelhante pode ser notado para o atributo $\theta_{\text {pmp }}$ ajustado por MQP.

Quando se confronta a Tabela 3 com a Tabela 4, percebe-se que os modelos de semivariogramas que apresentaram os maiores graus de dependência não repetiram o mesmo desempenho na validação cruzada, 
Tabela 3 - Parâmetros de ajuste dos modelos de semivariogramas e respectivos graus de dependência espacial.

\begin{tabular}{|c|c|c|c|c|c|c|}
\hline \multirow{2}{*}{ Atributos } & \multirow{2}{*}{ Método } & \multirow{2}{*}{ Modelo } & \multicolumn{3}{|c|}{ Parâmetros } & \multirow{2}{*}{$\mathrm{GD}(\%)$} \\
\hline & & & $\mathrm{C}_{\mathrm{o}}$ & $\mathrm{C}_{0}+\mathrm{C}$ & $\mathrm{a}(\mathrm{m})$ & \\
\hline \multirow{3}{*}{$\theta_{\mathrm{cc}}$} & \multirow{3}{*}{ MQP } & Esf & 23,72 & 36,3459 & 1516 & 35 \\
\hline & & Exp & 24,06 & 41,7764 & 1259 & 42 \\
\hline & & Gaus & 25,48 & 37,0827 & 831 & 31 \\
\hline \multirow{6}{*}{$\mathrm{k}_{\mathrm{o}}$} & \multirow{3}{*}{ MQP } & Esf & 0,71 & 1,1961 & 2798 & 40 \\
\hline & & Exp & 0,70 & 1,385 & 2000 & 50 \\
\hline & & Gaus & 0,76 & 1,1957 & 1316 & 36 \\
\hline & \multirow{3}{*}{ MV } & Esf & 0,56 & 1,1195 & 1588 & 50 \\
\hline & & Exp & 0,56 & 1,6282 & 2000 & 65 \\
\hline & & Gaus & 0,61 & 1,0755 & 640 & 43 \\
\hline \multirow{3}{*}{ CTA } & \multirow{3}{*}{ MQP } & Esf & 31,21 & 108,0201 & 1301 & 71 \\
\hline & & Exp & 27,61 & 125,2784 & 749 & 78 \\
\hline & & Gaus & 41,05 & 109,643 & 663 & 63 \\
\hline \multirow{6}{*}{$\theta_{\mathrm{pmp}}$} & \multirow{3}{*}{ MQP } & Esf & 7,26 & 12,9357 & 2154 & 44 \\
\hline & & Exp & 7,14 & 14,4455 & 1397 & 51 \\
\hline & & Gaus & 8,02 & 13,0977 & 1109 & 39 \\
\hline & \multirow{3}{*}{ MV } & Esf & 8,37 & 13,685 & 1500 & 39 \\
\hline & & Exp & 2,82 & 11,892 & 53 & 76 \\
\hline & & Gaus & 8,95 & 11,648 & 413 & 23 \\
\hline \multirow{3}{*}{ VTP } & \multirow{3}{*}{ MQP } & Esf & 6,62 & 40,9534 & 580 & 84 \\
\hline & & Exp & 4,08 & 42,2858 & 255 & 90 \\
\hline & & Gaus & 12,27 & 41,2492 & 309 & 70 \\
\hline \multirow{6}{*}{$\mu$} & \multirow{3}{*}{ MQP } & Esf & 61,69 & 84,4303 & 1400 & 27 \\
\hline & & Exp & 58,97 & 86,2906 & 583 & 32 \\
\hline & & Gaus & 64,95 & 84,7337 & 715 & 23 \\
\hline & \multirow{3}{*}{ MV } & Esf & 63,48 & 82,46 & 1000 & 23 \\
\hline & & Exp & 61,36 & 80,89 & 305 & 24 \\
\hline & & Gaus & 63,17 & 81,17 & 282 & 22 \\
\hline
\end{tabular}

$\theta_{\text {cc }}$ : umidade volumétrica na capacidade de campo; $\mathrm{k}_{0}$ : condutividade hidráulica do solo saturado; CTA: capacidade total de água na camada de 0 a $0,15 \mathrm{~m} ; \theta_{\mathrm{pmm}}$ : umidade volumétrica no ponto de murcha permanente; VTP: volume total de poros e $\mu$ : porosidade drenável; respectivamente.

produzindo os maiores erros médios padronizados e desvio padrão dos erros quando comparados aos demais modelos para o mesmo atributo, principalmente para o modelo exponencial ajustado por MQP para os atributos CTA e VTP.

A única exceção foi o atributo $\theta_{\text {pmp }}$, que apresentou o menor erro e desvio padrão dos erros com o modelo exponencial ajustado por MV, o qual apresentou o maior grau de dependência. Este fato revela que o GD não deve ser usado como parâmetro único na escolha de semivariogramas, pois apresenta um caráter subjetivo.

Com base nos valores das Tabelas 3 e 4, adotou-se para este trabalho o modelo gaussiano ajustado por mínimos quadrados ponderados para estudo da 
Tabela 4 -Erro médio reduzido ( $\overline{\mathrm{ER}})$, desvio padrão dos erros reduzidos $\left(\mathrm{S}_{\mathrm{ER}}\right)$ e somatório do quadrado médio do erro ( $\sum$ QME ), gerados pela validação cruzada.

\begin{tabular}{|c|c|c|c|c|c|}
\hline Atributos & Método & Modelo & $\overline{E R}$ & $S_{E R}$ & $\sum Q M E$ \\
\hline \multirow{3}{*}{$\theta_{\mathrm{cc}}$} & \multirow{3}{*}{ MQP } & Esf & 0,00248 & 1,00668 & 0,000321 \\
\hline & & Exp & 0,00254 & 0,98620 & 0,000256 \\
\hline & & Gaus & 0,00146 & 1,03874 & 0,000232 \\
\hline \multirow{6}{*}{$\mathrm{K}_{\mathrm{o}}$} & \multirow{3}{*}{ MQP } & Esf & $-0,00057$ & 0,92697 & 0,6581 \\
\hline & & Exp & $-0,00067$ & 0,93450 & 0,6235 \\
\hline & & Gaus & $-0,00001$ & 0,94012 & 0,5869 \\
\hline & \multirow{3}{*}{ MV } & Esf & $-0,00038$ & 0,99843 & 0,6587 \\
\hline & & Exp & $-0,00062$ & 0,99867 & 0,7564 \\
\hline & & Gaus & 0,00053 & 1,00055 & 0,6124 \\
\hline \multirow{3}{*}{ CTA } & \multirow{3}{*}{ MQP } & Esf & 0,00711 & 1,15283 & 3,21547 \\
\hline & & Exp & 0,00767 & 1,16543 & 3,35649 \\
\hline & & Gaus & 0,00336 & 1,13061 & 2,98745 \\
\hline \multirow{6}{*}{$\theta_{\mathrm{pmp}}$} & \multirow{3}{*}{ MQP } & Esf & 0,00104 & 1,11407 & 0,005123 \\
\hline & & Exp & 0,00098 & 1,13101 & 0,005231 \\
\hline & & Gaus & 0,00080 & 1,12529 & 0,004185 \\
\hline & \multirow{3}{*}{ MV } & Esf & 0,00115 & 1,01483 & 0,004521 \\
\hline & & Exp & $-0,00162$ & 1,01216 & 0,004212 \\
\hline & & Gaus & 0,00107 & 1,04069 & 0,004356 \\
\hline \multirow{3}{*}{ VTP } & \multirow{3}{*}{ MQP } & Esf & $-0,01203$ & 1,12443 & 0,000124 \\
\hline & & Exp & $-0,01520$ & 1,17842 & 0,000256 \\
\hline & & Gaus & $-0,01310$ & 1,14540 & 0,000245 \\
\hline \multirow{6}{*}{$\mu$} & \multirow{3}{*}{ MQP } & Esf & 0,00068 & 1,02061 & 0,006589 \\
\hline & & Exp & 0,00092 & 1,024284 & 0,006235 \\
\hline & & Gaus & 0,00081 & 1,031371 & 0,006479 \\
\hline & \multirow{3}{*}{ MV } & Esf & 0,00081 & 1,004114 & 0,006548 \\
\hline & & Exp & 0,00069 & 1,003384 & 0,006235 \\
\hline & & Gaus & 0,00061 & 1,004082 & 0,005687 \\
\hline
\end{tabular}

variabilidade espacial na sub-bacia hidrográfica para os atributos $\mathrm{k}_{\mathrm{o}}$ e CTA e o modelo esférico para os atributos VTP e $\theta_{\text {cc }}$, com a mesma metodologia. A metodologia da máxima verossimilhança é aplicada aos atributos $\mu$ e $\theta_{\text {pmp }}$, por meio dos modelos gaussiano e exponencial, respectivamente.

\section{CONCLUSÕES}

- A análise variográfica mostrou que todos os atributos estudados apresentaram-se estruturados espacialmente, sendo a maioria com grau moderado a forte de dependência espacial.

- Em termos de ajuste dos semivariogramas, a metodologia dos mínimos quadrados ponderados apresentou melhor performance, excetuando-se a porosidade drenável $(\mu)$ e umidade correspondente ao ponto de murcha de permanente $\left(\theta_{\text {pmpp }}\right)$.

- Em termos de modelos de semivariogramas, sugerese o modelo gaussiano para os atributos capacidade total 
de armazenamento (CTA), condutividade hidráulica saturada $\left(\mathrm{k}_{\mathrm{o}}\right)$ e porosidade drenável $(\mu)$; o esférico para os atributos umidade à capacidade de campo $\left(\theta_{c c}\right)$ e volume total de poros (VTP) e o exponencial para umidade no ponto de murcha permanente $\left(\theta_{\mathrm{pmp}}\right)$.

\section{REFERÊNCIAS BIBLIOGRÁFICAS}

BERNARDO, S. Manual de irrigação. 7. ed. Viçosa: UFV, 2005. 656 p.

BRUNDSDON, C.; FOTHERINGHAM, A. S.; CHALTON, M. E. Geographically weighted regression: a method for exploring spatial nonstationarity. Geographical Analysis, Columbus, v. 28, n. 4, p. 281-298, 1996.

CRESSIE, N. A. C. Statistic for spatial data. New York: J. Wiley, 1993. $900 \mathrm{p}$.

DIGGLE, P. J.; RIBEIRO JUNIOR, P. J. Model-based geostatistics. Londres: Springer Series in Statistics, 2007. $230 \mathrm{p}$.

GOMES, N. M. Variabilidade espacial de atributos físicohídricos do solo da sub-bacia hidrográfica do Ribeirão Marcela na Região do Alto Rio Grande, MG. 2005. 124 f. Dissertação (Mestrado) - Universidade Federal de Lavras, Lavras, 2005.

LAMPARELLI, R. A. C.; ROCHA, J. V.; BORGHI, E. Geoprocessamento e agricultura de precisão: fundamentos e aplicações. Guaíba: Agropecuária, 2001. 118 p.

LIBARDI, P. L.; MELO FILHO, J. F. Análise exploratória e variabilidade dos parâmetros da equação da condutividade hidráulica, em um experimento de perfil instantâneo. Revista Brasileira de Ciência do Solo, Viçosa, v. 30, n. 2, p. 197 206, 2006.

McBRATNEY, A. G.; WEBSTER, A. G. Choosing functions for semivariograms and fitting them to sampling estimates.
Journal of Soil Science, London, v. 37, n. 4, p. 617-639, 1986.

MEDINA, B. F.; OLIVEIRA JÚNIOR, R. C. Relações entre capacidade de campo determinada in situ e em laboratório em Latossolo Amarelo muito argiloso. Revista Brasileira de Ciência do Solo, Viçosa, v. 11, n. 1, p. 91-95, 1987.

MELLO, C. R. de; OLIVEIRA, G. C.; FERREIRA, D. F.; LIMA, J. M. Predição da porosidade drenável e disponibilidade de água para Cambissolos da microrregião Campos das Vertentes, MG. Pesquisa Agropecuária Brasileira, Brasília, v. 37, n. 9, p. 1319-1324, 2002.

MELLO, J. M.; BATISTA, J. L. F.; OLIVEIRA, M. S.; RIBEIRO JÚNIOR, P. J. Ajuste e seleção de modelos espaciais de semivariogramas visando a estimativa volumétrica de Eucalyptus grandis. Scientia Forestalis, Piracicaba, v. 69, n. 1, p. 25-37, dez. 2005.

RIBEIRO JUNIOR, P. P.; DIGGLE, P. P. GeoR: a package for geostatistical analysis. R - News, Auckland, v. 1, n. 2, p. 1518, 2001.

VIEIRA, V. A. S. Variabilidade espacial da estabilidade de agregados do solo em uma microbacia hidrográfica. 2005. 100 p. Dissertação (Mestrado) - Universidade Federal de Lavras, Lavras, 2005.

WARRICK, A. W.; NIELSEN, D. R. Spatial variability of soil physical properties in the field. In: HILLEL, D. (Ed.). Applications of soil physics. New York: Academic, 1980. p. 319-344.

ZIMBACK, C. R. L. Análise espacial de atributos químicos de solos para fins de mapeamento da fertilidade. 2001. 114 f. Tese (Livre-Docência) - Universidade Estadual de São Paulo, Botucatu, 2001. 